\title{
ASSIST - Automated System for Surgical Instrument and Sponge Tracking
}

\author{
Nilo Rivera $^{1}$, Rosemary Mountain ${ }^{1}$, Lia Assumpcao ${ }^{2}$, Allen A. Williams ${ }^{1}$, A.B. Cooper ${ }^{1}$, \\ Douglas L. Lewis ${ }^{3}$, Richard C. Benson ${ }^{3}$, Joseph A. Miragliotta ${ }^{3}$, Mike Marohn $^{2}$, and Russell H. Taylor ${ }^{1}$ \\ ${ }^{1}$ Whiting School of Engineering, Johns Hopkins University, Baltimore, MD \\ 2 Department of Surgery, Johns Hopkins Medical Institution, Baltimore, MD \\ 3 Applied Physics Laboratory, Johns Hopkins University, Laurel, MD
}

\begin{abstract}
Every surgical item used during surgery (e.g., sponges) must be accounted for after surgery to ensure that none of these items is left inside the patient. Despite the numerous precautions in place, in approximately 1 in 1500 cases, something gets left behind inside the patient's body.

This paper presents ASSIST, an automated system for surgical instrument and sponge tracking that increases the safety of surgical procedures. ASSIST utilizes RFID (Radio Frequency Identification) technology to aid in accounting for all items used during surgery. The design takes into account safety, simplicity, ease of deployment, and ease of use. An initial evaluation utilizing RFID-tagged sponges demonstrates that ASSIST can reliably track surgical sponges with minimal impact to current operating room procedures. Sources of error that can impact the reliability of the system are also discussed.
\end{abstract}

\section{INTRODUCTION}

One dangerous medical error that can occur during surgery is unintentionally leaving a surgical instrument or sponge inside a patient. Commonly known as retained foreign object, this error can lead to inflammation, obstruction, perforation, sepsis, and sometimes death. The problem is thought to be avoidable when stringent manual counting guidelines are followed by Operating Room (OR) personnel[1]. While these guidelines are very effective in reducing the risk, the problem persists [2] [3] [4]. Some estimates report that the incidence can be as high as 1 in 1500 surgeries [5].

Human error is not the only drawback of manual counting. During sponge counting, nurses are unable to provide support for the surgeon as they are focused on accurately counting sponges. Each sponge count takes a couple of minutes, with at least three counts per surgical procedure. Under these counting procedures, the nurse is inevitably distracted from her primary role for a significant part of the time. Also, when a miscount is found, there is a significant increase in the OR time since an X-ray of the patient is many times required. Some hospitals $\mathrm{x}$-ray every patient after any opencavity operation, which requires a radiologist to be available after every surgery and unnecessarily exposes the majority of patients to radiation.

This paper presents ASSIST, an automated system for surgical instrument and sponge tracking that increases the safety of surgical procedures. With ASSIST, RFID (Radio Frequency Identification) technology is used to detect and uniquely identify each surgical item at various stages during surgery. The use of low frequency RFID enables reliable detection of tags even when soaked in body fluids, in the vicinity of metallic objects such as surgical tools, or inside a patient's body [6]. A software-based inventory component keeps track of every item, and enables users to quickly identify the state of the procedure through a color-coded interface.

While ASSIST can track any item tagged with an RFID device, our initial design focuses on retained sponges (a.k.a. gossypiboma) as they constitute the majority of retained foreign body cases. A check-in station verifies the content of a package and registers each tagged item in a database to keep an inventory. A check-out station, consisting of a smart bucket where sponges are discarded, accounts for used sponges. The number of items in-use is displayed to the user at all times. If zero, all items are accounted for. Otherwise, a patient scanner is available to detect whether a missing sponge is still inside the patient.

ASSIST has several benefits over current practice. First, it eliminates false-positives, i.e., counts that appear to be complete when a sponge is still missing, which are estimated to account for more than $80 \%$ of cases of retained foreign objects. Second, ASSIST eliminates unnecessary exposure to $\mathrm{X}$-rays as only those patients that appear to have unaccounted items need to be x-rayed. Third, it reduces the operational cost to the hospital by reducing nurse and OR time required for each intervention. Finally, real time information on the state of retained foreign objects allows the surgeon to close an incision without delay.

Our initial investigation shows that our system can reliably account for $100 \%$ of tagged sponges during surgery. This high level of reliability is attained by RFID verification during check-in, and continuous counting with multiple orthogonal antennas at the check-out bucket. The read rate is maximized by utilizing random antenna selection between consecutive reads. The measured check-in time for a 10sponge packet is just 2 seconds, while regular check-out time is between 1 to 5 seconds when several sponges are thrown into the bucket at the same time. Experiments also show that we can detect missing sponges inside an in vivo porcine model, with an RFID patient scanner, in less than 5 seconds. 


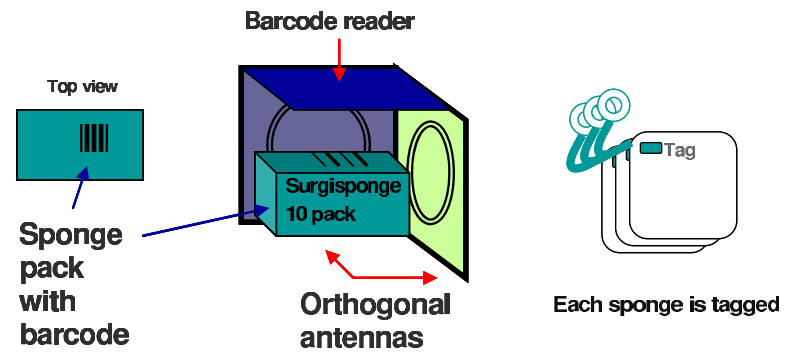

Fig. 1: Check-In Station

\section{BACKGROUND}

In healthcare, RFID has two major areas of application, administrative and direct-patient. Administrative applications include supply chain, smart shelving, and equipment and/or pharmaceuticals tracking. RFID technology is becoming well established in these areas due to efficiencies established in commercial and industrial sectors. Direct-patient applications, on the other hand, are still in their infancy largely because they can have a direct impact on the patient's health. One example is VeriChip[7], approved by the FDA in 2004, and offers implantable RFID chips containing personal medical information to help limit medical treatment errors. ASSIST will also require FDA approval as it directly affects patients' health and safety.

Two general variants of RFID technology exist; active tags, which require an internal power source, and passive tags, which rely on the incoming radio frequency signal to power up and respond to commands. Both tag systems operate in different frequencies; low, high, ultra high, and microwave. Low frequency (LF) tags work in the $125-148 \mathrm{KHz}$ range and utilize magnetic fields for communication. They are largely unaffected by the presence of fluids, non-ferrous metals, and most electromagnetic (EM) noise sources. High frequency (HF) tags work at $13.56 \mathrm{MHz}$, can read some distance through fluids, and are susceptible to noise and antenna detuning in the presence of metals. Higher frequency RFID devices utilize electric fields for communication which are attenuated by fluids and are therefore not suited for tracking fluid soaked sponges. Finally, there are two families of anticollision protocols: binary tree and Aloha. The binary tree provides a deterministic approach to read every available tag, while Aloha derived protocols are purely stochastic and rely on probability to ensure that every tag is read.

RFID is well suited for automating sponge counting in the OR. It allows us to uniquely identify each tag and reliably account for each one during surgery. We chose passive tags as they do not require an internal energy source, last much longer than active tags, can be made in much smaller sizes, and can be manufactured at a significantly lower cost. Low frequency tags enable us to search for items within a patient with high reliability as organs, bones, and body fluids are transparent to magnetic fields. In addition, we choose tags

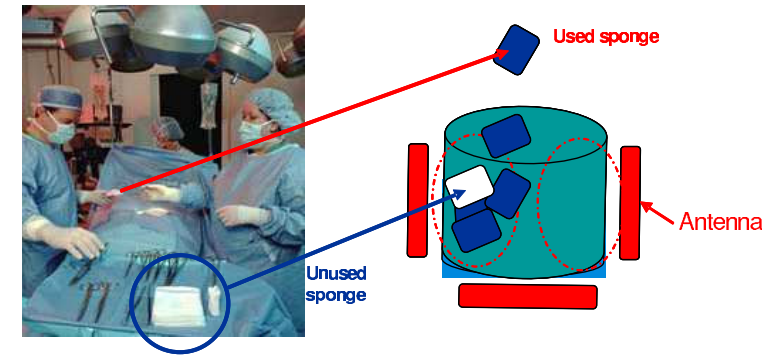

Fig. 2: Check-Out Station

that utilize a binary tree anticollision protocol as we need to account for every item before and after surgery with the highest certainty.

\section{APPROACH}

Our approach is to develop an electronic inventory that can keep track of every surgical item used during surgery. RFID is used for non-line-of-sight identification (a unique serial number for each sponge can be received by wireless means). A check-in station, a check-out station, and a patient scanner are used by OR personnel to track and/or find sponges throughout the surgery. All of these components are controlled via a software system that utilizes a color-coded interface for easy and fast assessment of the location of the items during surgery.

\section{A. Check-in Station}

The check-in station consists of two orthogonal RFID antennas surmounted on top by a perpendicular UPC barcode reader. A design is depicted in Figure 1. The check-in station is small, simple, and occupies very little space. The UPC barcode identifies a package and the number of items within it. Before accepting the package, ASSIST verifies that the package has the expected number of items in it, which is gathered from barcode information. If the package contains a bad RFID tag, the reader will recognize the discrepancy and subsequently direct the removal of the package. In addition, the barcode provides us with a description of the type of sponge associated with each RFID tag. Note that this barcode can be easily replaced by an RFID-based Electronic Product Code (EPC) [8].

It is also possible to store the barcode information in the RFID tags already available inside the sponges. While this is possible, it would increase the price of the tags as they would require read/write memory to hold this information instead of just a unique read-only ID. In addition, sponge manufacturers would have to ensure that the RFID tags that they attach to the sponges are programmed with the correct information. Further, the same sponge may be packaged in different quantities, which requires different codes to be inserted in tags that are destined for different packages. As such, it is simpler to attach that information to the packaging means of a barcode or EPC. 


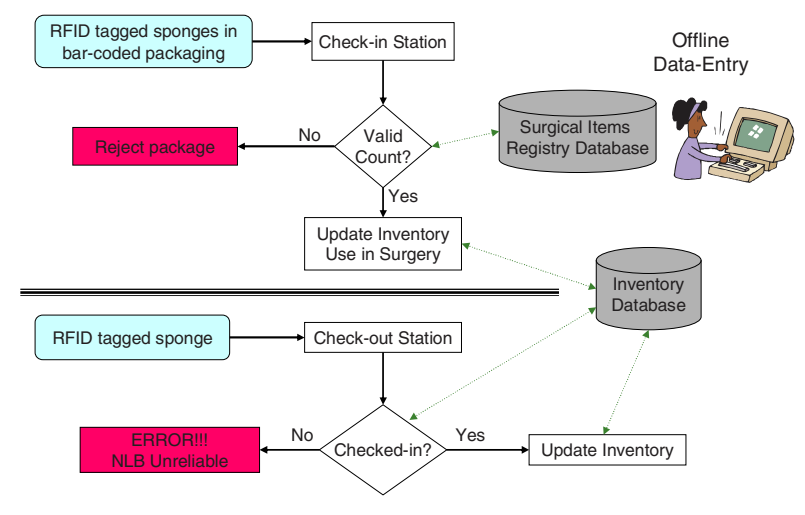

Fig. 3: Control Flow Overview

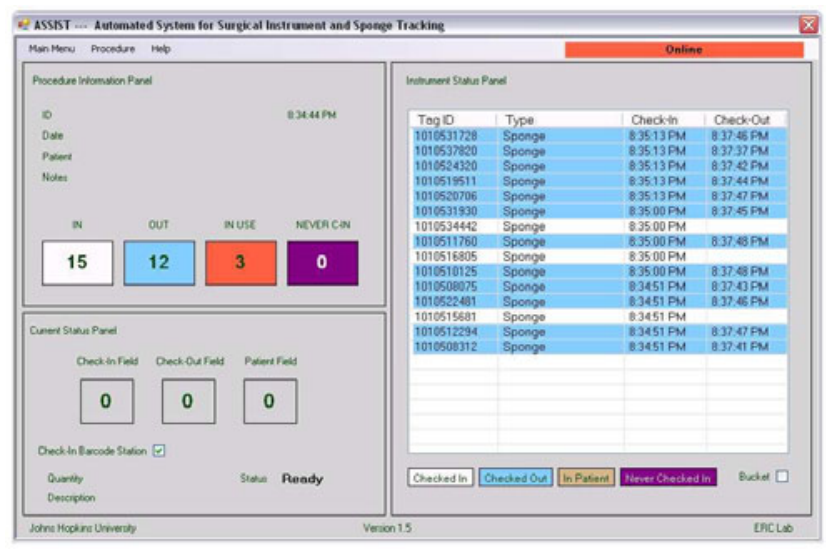

Fig. 4: GUI
When a package is accepted, every item is registered into the system inventory where it can be tracked throughout the surgery. As previously stated, each sponge is distinguishable by its RFID tag which has a unique serial number.

\section{B. Check-out Station}

A stainless-steel bucket, commonly known as a kickbucket, is the normal depository for used sponges in the OR. It is small, simple, and convenient. Our approach is to provide a new kickbucket with a similar form factor, enabling the OR to replace existing ones with minimum disruption.

The ASSIST kickbucket is equipped with an RFID reader, five orthogonal antennas (four sides plus the bottom), and the means to talk to the software component that keeps track of the inventory (i.e. wireless serial port). Since every RFID tag is equipped with a unique serial number, no sponge is counted more than once. A design is depicted in Figure 2.

The ASSIST kickbucket continuously scans for sponges and updates the inventory accordingly. While 5 antennas may seem excessive, our initial results suggest that we require all of them to ensure that all the sponges are read reliably without any significant involvement from OR personnel. Further design refinement may result in a more economical design, but such considerations are left for a subsequent design phase.

Although the ASSIST kickbucket is intended for use throughout the surgery, the OR staff can also use another, non-RFID equipped kickbucket to dispose sponges, and transfer them all (possibly in a bag) into the ASSIST kickbucket at the end of the surgical procedure.

\section{Patient Scanner}

When a sponge is considered "missing", current practice OR procedure dictates that an x-ray of the patient be taken. This requires an $\mathrm{x}$-ray machine to be brought into the OR, or that the patient be moved to an x-ray equipped location. A radiologist needs to be called to read the x-ray. The x-ray may not reveal the missing sponge, especially in cases where the sponge is likely to be surrounded by bones (e.g., heart surgery).

A patient scanner can be used to detect, and somewhat localize, missing sponges in the patient. We use a blanket embedded with a large antenna that allows us to reach deeper into the patient, increasing the chance of finding any missing sponge. Smaller antennas may also be embedded in the blanket to localize the missing sponge. Any sponge found by the patient scanner is identified as "In-Patient" in the software inventory.

While very convenient, some medical devices may react inappropriately to the presence of an RFID patient scanner. For example, a recent study by the FDA [9] shows that RFID can interfere with pacemakers and Implantable Cardiac Defibrillators. These patients can still benefit from ASSIST accurate inventory system, but must resolve to other means of finding missing sponges when necessary (e.g., x-ray).

\section{Software System}

It is routine for most OR facilities to utilize computer monitors during surgical procedures. Our approach is to incorporate our inventory software-based approach with the current OR computer infrastructure. In general, the software system should be multi-layered, extensible, and fault tolerant, enforcing when possible the policies in place to ensure compliance with the new procedure.

ASSIST uses two databases; the surgical items registry, and the inventory. The registry contains barcode information for each package (i.e. description, quantity). The inventory keeps track of all items that have been checked-in as they pass through the system and makes sure that every item is accounted for after the surgery is complete. Any missing item will be reflected in the inventory, at which time the patient must be scanned by the patient scanner and/or x-ray. An overview of the different databases and the flow of the procedure is shown in Figure 3.

The Graphical User Interface ties together all the different components of the system. A snapshot of the ASSIST GUI 


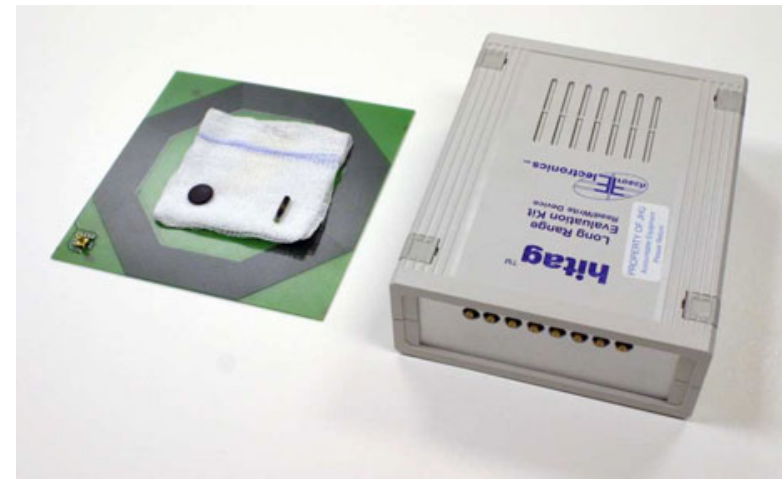

Fig. 5: Equipment

\begin{tabular}{|c|r|r|}
\hline \hline Antennas & One Cycle & Five Cycles \\
\hline 1 & $63.9 \%$ & $71.7 \%$ \\
2 & $74.7 \%$ & $91.7 \%$ \\
3 & $84.4 \%$ & $94.4 \%$ \\
4 & $91.4 \%$ & $98.6 \%$ \\
5 & $97.2 \%$ & $100.0 \%$ \\
\hline
\end{tabular}

TABLE I: Number of antennas required to reliably find 40 sponges in check-out bucket.

can be seen in Figure 4. It contains three-panels, each with color-coded identifiers which allow OR staff to quickly and easily identify any problem and assess the state of the procedure.

\section{EXPERIMENTAL RESULTS}

Our prototype system comprises of a Frosch Electronics Low Frequency RFID reader with a built-in multiplexer, seven $18 \times 18 \mathrm{~cm}$ PCB antennas, a $30 \times 40 \mathrm{~cm}$ flat cable ribbon antenna for the patient scanner, a Symbol OmniDirectional barcode scanner, and approximately 100 passive low-frequency hitag 1/S RFID tags. Figure 5 shows some of the parts used in our experiments. All RFID tags where glued inside Kendall 4x4 raytec sponges (one in each).

Two PCB antennas were arranged orthogonal to one another in the check-in station, with the barcode scanner on top. The other five PCB antennas were arranged around the sides of the ASSIST kick-bucket, while leaving the top open. Tag orientations inside the kick-bucket are random. Mutual inductance between tags that are in close proximity can change their frequency response and make them unresponsive to the tag reader. The packaged sponges can be arranged in such a way that they do not interfere with one another but there is no such control over the position of the used sponges in the bucket.

Our initial experiments examine the reliability and feasibility of the system using dry sponges with RFID tags. First, we identified the number of antennas required to reliably detect sponges in the check-out bucket. We deposited 40 tagged sponges in the bucket, and noted the number of sponges found when using different numbers of antennas and

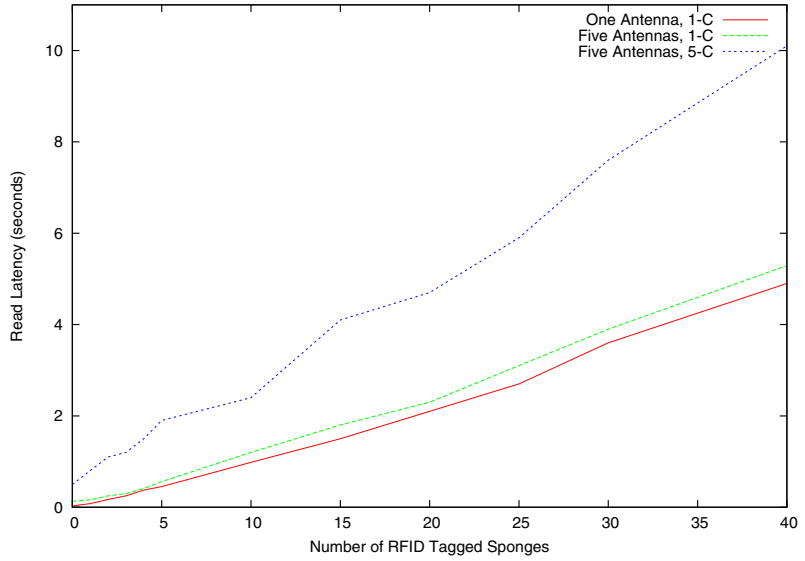

Fig. 6: Time to read sponges with one antenna, 5 antennas, and 5 cycles with 5 antennas

performing one or multiple read cycles. A read cycle consists of one read from each of the antennas, so five cycles with one antenna is identical to reading five consecutive times from the same antenna. The experiment was repeated 20 times, revolving the sponges each time so that they ended up in different positions. Table I shows that we required all five antennas, and multiple read cycles, to reliably detect all sponges in the bucket.

Reading from five antennas multiple times will introduce some unavoidable latency. Figure 6 shows how the read latency increases as more sponges are deposited in the bucket ${ }^{1}$. Reading from multiple antennas resulted in less than $10 \%$ (1/2 second) additional latency. The overhead is low because the anti-collision procedure turns off tags as it reads them, and so consecutive reads from other antennas will find a smaller set of tags to read from. Performing 5 cycles introduces another few seconds of latency. As we are using a multiplexer, only one antenna at a time can send the refresh signal to make sure that the tags stay in silent mode. Tags that are not aligned with that antenna do not hear this signal and so they turn on again, and are read again by another antenna in consecutive cycles.

The system was then tested on a more realistic setting at the Johns Hopkins Minimally Invasive Surgery Training Center (MISTIC) center. ASSIST was used in five in-vivo pig surgeries, on three different sessions. Each operation consisted of an open abdominal and/or thoracic cavity surgery where organs were explored, and sometimes extracted, as they would be in a regular surgery. RFID tagged sponges where used in each intervention. Sponges were deliberately left inside the "patient" after surgery, and the system correctly determined the number of sponges missing each time.

\footnotetext{
${ }^{1}$ Using one antenna could not reliably read the complete pool of sponges as the RFID tags lied in random orientations with only a subset appropriately aligned to the field lines of a single antenna at any one time. We had to redistribute the sponges several times in order to read all tags in one cycle.
} 


\begin{tabular}{|c|r|r|}
\hline Test & Detail & Result \\
\hline Time to check-in & 10 sponge pack & 2 seconds \\
\hline Time to find 7 sponges thrown & Empty Bucket & 2 seconds \\
into bucket as bucket gets & 25 sponges & 3 seconds \\
filled (online reading) & 45 sponges & 6 seconds \\
& 60 sponges & 10 seconds \\
\hline Time to find all sponges & 25 sponges & 6 seconds \\
simultaneously in the bucket & 45 sponges & 12 seconds \\
(offline reading) & 60 sponges & 31 seconds \\
\hline
\end{tabular}

TABLE II: Time that ASSIST takes to detect and account for sponges in different scenarios

We then performed a series of "hide-and-seek" tests where one person hid sponges in different locations within the animal, and another looked for the missing sponges with the patient scanner. Sponges where hidden around the stomach, in the pelvic hole, and behind the sternum and ribs, including areas around the heart. On each occasion, all of the missing sponges were successfully found in less than 5 seconds of scanning. Reverse experiments were also performed, where multiple $(>5)$ sponges were left in the patient around the abdomen and the chest, and the patient scanner was used to find all sponges before a count was performed in the bucket, i.e., the person scanning did not know how many sponges were missing. Each time, the final count of the bag revealed all sponges were successfully found without having to rescan the patient.

Once the animals were euthanized, we collected enough blood to test the reliability of the system after the sponges were immersed in blood. This way, we could perform a realistic evaluation of the reliability of the system under realistic surgical conditions (bearing in mind that wireless signals can be affected by fluids and metals). A different number of blood-soaked sponges were used on three different occasions: 25,45 , and 60 . The sponges were redistributed randomly on each trial.

We found that, with one cycle through all five antennas, the detection rate was about $95 \%$. With multiple (5) repeated cycles the detection rate was between $98 \%$ and $100 \%$. While dry sponges did achieve $100 \%$ detection rate, the bloodsoaked ones were not always all found. One solution was to agitate the sponges (i.e. shake the bag). We found that a small movement of the bag quickly resulted in $100 \%$ detection rate. However, we also found that randomly selecting the antenna between consecutive reads produced $100 \%$ read rate, without having to move the sponges. This finding basically reflects the benefit of anticollision from adjacent reads.

Table II shows a summary of what a user would experience when using our system. The average time to check-in a sponge packet is just under 2 seconds. The average time to check-out sponges varies depending on the number of sponges in the bucket, with a steep increase in latency when 60 sponges were inside the bucket.

Finally, we performed experiments where metal instruments were "inadvertently" dropped in the bucket (a few surgical scissors). We had to move the sponges on 2 occa- sions, out of 15 tries, to be able to detect all sponges in the bucket. However, while it did take some additional effort to find them, all of the sponges were reliably, and accurately, accounted for by the ASSIST system.

\section{SOURCES OF ERROR}

ASSIST provides an alternate mechanism to keep track of surgical sponges during surgery. Several key advantages exist over current practice, including no false-positives, the ability to eliminate false-negatives, time savings, added confidence, and elimination of unnecessary x-ray exposure. However, as with other systems[10], ASSIST is susceptible to a variety of issues. While the likelihood of some of these conditions occurring is very small, it is important to be aware and understand what can go wrong.

- Protocol violation: An item that was never checkedin may be used during surgery, at which point the final count cannot be trusted. We can distinguish any unchecked item at check-out and report the problem. The nurse must identify the number of sponges that are likely to have been missed during check-in (i.e. typical quantity in package) and make sure the NeverChecked-In count in the ASSIST software reflects this number. The patient should also be scanned with a patient scanner and/or x-ray.

- Damaged RFID Tag: Any damaged RFID tag will not be able to be checked-in by the system. The barcode scanner prevents this type of error from becoming a problem as it will make sure that all of the tags are readable before they are used in surgery. However, there is a finite probability that a tag that is working when checked-in stops working during the procedure. This will result in a false negative. In this case, a manual count can be performed to verify the number of checked-out sponges.

- Unreliable Manufacturing Process: While ASSIST can detect and/or race a flag for single-errors like a damaged RFID tag, double-errors can occur in an unreliable manufacturing process. For example, if a 10-pack of sponges contains 11 sponges, and one of these sponges is either untagged or with a damaged tag, ASSIST will accept the package, but only 10 sponges will be entered into the inventory. While extremely unlikely (as it requires that there is a packaging error and that one of the RFIDs in the package is damaged or missing), we should be aware of the possibility as this kind of error can ultimately result in an unreliable system. One possible solution is to have the OR nurse manually count sponges, during check-in, to verify the expected quantity in the box.

- Computer and software failure: The system may crash or lose its ability to continue operating due to a hardware failure, corrupted memory, or software bug. One solution is to replicate the inventory to a backup system 
as the surgery progresses, enabling the surgical staff to access the information if needed. Another approach is to have an online printer that prints the status of the inventory in real time, during surgery, as sponges are checked-in and/or checked-out. Both approaches allow OR staff to revert to manual counting when the system is unable to proceed.

\section{RElATED WORK}

A recent case-control study[5] showed that the risk of retained foreign objects increases during emergencies, unplanned changes in procedure, and in patients with high body-mass index. However, more astonishingly, they show that $88 \%$ of analyzed cases involved a final count that was erroneously thought to be correct (false positive). Other case control studies show a similar trend [3] [2] [11].

A variety of approaches have been proposed to mitigate the problem of retained foreign objects following surgery. One proposal is the use of sequentially numbered surgical sponges[12], to reduce human-error during manual counting. This method is simple and does not significantly change the current procedure. Another approach, patented by Surgicount Medical, uses a printed barcode on each sponge with a unique identification number. This requires nurses to manually check-in and check-out every sponge with a lineof-sight barcode reader, a process that must be made more difficult by the presence of blood.

Others have looked at the problem of finding sponges inside a patient. In one approach, an electronic tag is inserted in every sponge [13]. As in our approach, they choose a LF (in their case 50 to $70 \mathrm{kHz}$ ) tags. Six sponges were hidden in the abdomen and the thorax of 50 cadavers weighting between 45 and $190 \mathrm{~kg}$, and $100 \%$ of the tags where found. Another low frequency solution, at $145 \mathrm{KHz}$, is commercially marketed by RF Surgical Systems. Note that these systems lack unique identification means as in RFID, therefore, they cannot provide an accurate inventory of the surgical instruments before and after the procedure.

Another study [14] evaluated the use of a wand to find high frequency (HF, 13.56MHz) RFID tagged sponges in 8 patients. A sponge was hidden in the abdomen of each patient. The RFID wand reliably detected each of the sponges, within five seconds. However, more studies are needed with HF RFID to validate its use for detecting sponges inside patients. High frequency signals are attenuated by body fluids and can be obstructed by bone, so the depth of field is likely to be insufficient for patients with large body mass.

An alternate system has been proposed by Clearcount Medical Solutions [15]. In their approach, HF RFID tagged sponges are tracked using a stand-alone device that has a scanner on one side and a waste disposal bucket on the other. The sponges are detected as they pass into the bucket rather than being detected inside the bucket, which has potential for errors when many sponges are thrown in together. The high frequency tags can be read at a faster rate than low frequency ones which helps to ensure that all of the sponges are detected as they enter the bucket but the trade-off is poorer penetration of body fluids leading to difficulty detecting the sponges inside the patient and also when wadded together. As this is a stand-alone system it does not integrate into the OR software but simply offers the user information on how many sponges have been checked in and checked out. There is no published data available on this system, so it is not possible to compare it's reliability with ASSIST.

\section{CONCLUSION}

ASSIST provides the OR staff with a reliable and accurate record of the status of each surgical item throughout the surgical procedure. Human error is minimized as the system eliminates false-positive (mistakenly complete count) and reduces false-negatives (the mistaken belief that a sponge is missing). Also, we cut down the time and effort that is otherwise needed for manual counting during for each intervention. Sources of error were identified, and the system was evaluated under realistic surgical conditions.

\section{REFERENCES}

[1] "Recommended practices for sponge, sharp, and instrument counts aorn recommended practices committee. association of perioperative registered nurses.” AORN J, vol. 70, no. 6, pp. 1083-9, 1999.

[2] C. Kaiser, S. Friedman, K. Spurling, T. Slowick, and H. Kaiser, "The retained surgical sponge." Ann Surg, vol. 224, no. 1, pp. 79-84, 1996.

[3] A. Gonzalez-Ojeda, D. Rodriguez-Alcantar, H. Arenas-Marquez, E. Sanchez Perez-Verdia, R. Chavez-Perez, R. Alvarez-Quintero, and A. Perea-Sanchez, "Retained foreign bodies following intra-abdominal surgery." Hepatogastroenterology, vol. 46, no. 26, pp. 808-12.

[4] C. Zhan and M. Miller, "Excess length of stay, charges, and mortality attributable to medical injuries during hospitalization." JAMA, vol. 290, no. 14, pp. 1868-74, 2003

[5] A. Gawande, D. Studdert, E. Orav, T. Brennan, and M. Zinner, "Risk factors for retained instruments and sponges after surgery." $N$ Engl J Med, vol. 348, no. 3, pp. 229-35, 2003.

[6] K. Finkenzeller, RFID handbook: fundamentals and applications in contactless smart cards and identification, 2nd ed., 2003.

[7] M. Levine, B. Adiba, K. Mandl, I. Kohane, and J. Halamka, "What are the benefits and risks of fitting patients with radiofrequency identification devices." PLoS Medicine, vol. 4, no. 11, 2007.

[8] S. Sarma, D. Brock, and D. Engels, "Radio frequency identification and the electronic product code," IEEE Micro, vol. 21, no. 6, pp. 5054, 2001.

[9] S. Seidman, P. Ruggera, R. Brockman, B. Lewis, and M. Shein, "Electromagnetic compatibility of pacemakers and implantable cardiac defibrillators exposed to rfid readers," International Journal of RFID Technology and Applications, vol. 1, no. 3, 2007.

[10] A. Rogers, E. Jones, and D. Oleynikov, "Radio frequency identification (rfid) applied to surgical sponges." Surg Endosc, 2007.

[11] A. E. Lincourt, A. Harrell, J. Cristiano, C. Sechrist, K. Kercher, and B. T. Heniford, "Retained foreign bodies after surgery." The Journal of surgical research, vol. 138, no. 2, pp. 170-174.

[12] M. Pelter, K. Stephens, and D. Loranger, "An evaluation of a numbered surgical sponge product.” AORN J, vol. 85, no. 5, pp. 931-40, 2007.

[13] C. Fabian, "Electronic tagging of surgical sponges to prevent their accidental retention.” Surgery, vol. 137, no. 3, pp. 298-301, 2005.

[14] A. Macario, D. Morris, and S. Morris, "Initial clinical evaluation of a handheld device for detecting retained surgical gauze sponges using radiofrequency identification technology." Arch Surg, 2006.

[15] "ClearCount Medical Solutions." [Online]. Available: http://www.clearcount.com 